\title{
Selecting and Utilizing Sequential Residual Generators in FDI Applied to Hybrid Vehicles
}

Christofer Sundström, Erik Frisk and Lars Nielsen

\section{Linköping University Post Print}

\section{Tweet}

N.B.: When citing this work, cite the original article.

(C2009 IEEE. Personal use of this material is permitted. However, permission to reprint/republish this material for advertising or promotional purposes or for creating new collective works for resale or redistribution to servers or lists, or to reuse any copyrighted component of this work in other works must be obtained from the IEEE.

Christofer Sundström, Erik Frisk and Lars Nielsen, Selecting and Utilizing Sequential Residual Generators in FDI Applied to Hybrid Vehicles, 2014, IEEE TRANSACTIONS ON SYSTEMS MAN CYBERNETICS-SYSTEMS, (44), 2, 172-185.

http://dx.doi.org/10.1109/TSMC.2013.2248147

Postprint available at: Linköping University Electronic Press http://urn.kb.se/resolve?urn=urn:nbn:se:liu:diva-104283 


\title{
Selecting and Utilizing Sequential Residual Generators in FDI Applied to Hybrid Vehicles
}

\author{
Christofer Sundström, Erik Frisk, and Lars Nielsen
}

\begin{abstract}
For a realistic model of a complex system there will be thousands of possible residual generators to be used for diagnosis. Based on engineering insights of the system to be monitored, certain algebraic and dynamic properties of the residual generators may be preferred, and therefore a method for finding sequential residual generators has been developed that accounts for these properties of the residual generator candidates. It is shown that only a small fraction of all residual generator candidates fulfill fundamental requirements, and thereby proves the value of systematic methods. Further, methods are devised for utilization of the residual generators, such as initialization of dynamic residual generators. A proposed method, considering the fault excitation in the residuals using the internal form of the residuals, significantly increases the diagnosis performance. A hybrid electric vehicle is used in a simulation study for demonstration, but the methods used are general in character and provides a basis when designing diagnosis systems for other complex systems.
\end{abstract}

\section{INTRODUCTION}

Design of a diagnosis system for a non-trivial real application is a complex engineering task involving many aspects [1], [2], [3]. Physical and semi-physical modeling will typically lead to a set of equations where some are algebraic and some are differential equations. Based on such a model of differential-algebraic equations (DAE), it is typically possible to construct many residuals to be used for fault detection and isolation [1]. How to select which of these residual generators to use is a non-trivial task, and further, before a diagnostic decision is made the residuals are often post-processed to form test quantities, e.g., by using the well-known CUSUM [4] algorithm.

When hybridizing a vehicle [5], new components are added compared to a conventional vehicle, e.g. electric machines, battery, and power electronics. It is important to monitor these components due to safety issues and to avoid damaging components, especially the battery that is sensitive and costly. From the viewpoint of needed methodology for diagnosis design, it is fundamental that these systems by their very nature switch between combustion engine and electrical motor, and under these two main modes there are many sub-modes. This may typically result in that different residuals are switched on and off, resulting in design questions on e.g. reinitialization when being turned on.

The contributions in the paper concerns the selection of the residual generators to be used in the diagnosis system, and methods for utilizing these residual generators to make diagnostic decisions. The method used for generating residuals

C. Sundström, E. Frisk, and L. Nielsen are with the Dept. of Electrical Engineering, Linköping University, SE-581 83 Linköping, Sweden $\{$ csu,frisk,lars\}@ isy.liu.se is based on computation sequences of the unknown variables [6], and is called sequential residual generators by [7]. To this method, one would like to add engineering insights where intuition regarding noise properties may make a dynamic computation sequence leading to integration preferable compared to a sequence resulting in differentiation. Different computation sequences leads to different results regarding algebraic loops and uniqueness, which is exploited in the diagnosis design. Furthermore, in non-linear systems the fault excitation often depends on the operating mode of the system to be monitored, and a method for exploiting this when designing the test quantities is proposed. The method, that is one of the main contributions in the paper, is based on the internal form of the residual generators and is exemplified for the CUSUM algorithm. Finally, all these aspects, i.e. the investigation of all sequential residual generator candidates and utilization of a set of these, are illustrated on an industrial example in a simulation study for a hybrid electric vehicle.

\section{RESIDUAL GENERATOR CONSTRUCTION}

The objective of this section is to introduce, and make slight modifications to, the model based residual generation technique that is analyzed further in the following sections. Examples of proposed techniques to generate residuals are parity equations [8], variable elimination [9], parameter estimation [10], and state-observer [11] techniques. As stated above, sequential residual generators are used here and the basic principle is straightforward [6], [7]. A sequential residual generator consists of a set of equations with analytical redundancy where a subset of the equations are used to solve for the unknown variables included in these equations, and then the redundant equations are used to check consistency between the observations and the set of model equations. The basic principle is illustrated with a small example.

Example 1. Consider the model

$$
\begin{aligned}
& e_{1}: x_{1}=g_{1}\left(x_{2}\right) \\
& e_{2}: x_{2}=g_{2}(u) \\
& e_{3}: y=x_{1}
\end{aligned}
$$

with 3 equations, 2 unknown variables $x_{1}$ and $x_{2}$, known input variable $u$, measurement variable $y$, and non-linear functions $g_{i}$. Here, both $e_{1}$ and $e_{3}$ can be chosen as the consistency relation, or analytical redundancy relation (ARR) [9], resulting in the two residual generators

$$
\begin{array}{ll}
x_{2}:=g_{2}(u) & x_{2}:=g_{2}(u) \\
x_{1}:=y & x_{1}:=g_{1}\left(x_{2}\right) \\
r_{1}:=x_{1}-g_{1}\left(x_{2}\right) & r_{2}:=y-x_{1} .
\end{array}
$$


respectively.

In the simple example above, the different choice of consistency relation resulted in equivalent residual generators. However, in general the choice may strongly affect the performance of the diagnosis system, which is shown in Sections IV and VI using the hybrid vehicle as an example.

\section{A. Sequential Residual Generation by Structural Analysis}

This presentation will be based on the notation in [7] and a brief outline of the approach is included here. The system model is denoted by $M(E, X, Z)$ where $E=\left\{e_{1}, e_{2}, \ldots, e_{m}\right\}$ is the set of equations, $X=\left\{x_{1}, x_{2}, \ldots, x_{n}\right\}$ the set of unknown variables, and $Z=\left\{z_{1}, z_{2}, \ldots, z_{r}\right\}$ the set of known variables. It is assumed that the set of equations is expressed as a semi-explicit DAE, i.e.

$$
\begin{aligned}
\dot{x}_{d} & =f\left(x_{d}, x_{a}, z\right) \\
0 & =g\left(x_{d}, x_{a}, z\right)
\end{aligned}
$$

where $z$ is a vector of known variables and $x_{d}$ are the dynamic variables and $x_{a}$ the algebraic variables, both vectors of unknown variables $x_{i}$. The semi-explicit form leads to that no more than one differentiated variable is included in each equation.

In accordance with the example above, a sequential residual generator is based on a set of equations $\bar{E} \subseteq E$ with analytical redundancy where the set of unknown variables in $\bar{E}$ is denoted by $X^{\prime} \subseteq X$. In this presentation, subset minimal sets of equations with structural redundancy 1 will be considered, i.e., $|\bar{E}|=\left|X^{\prime}\right|+1$ and no proper subset $\tilde{E} \subsetneq \bar{E}$ has structural redundancy $>0$. A sequential residual generator based on $M\left(\bar{E}, X^{\prime}, Z\right)$ then consists of a consistency relation $e_{i} \in \bar{E}$ and the just-determined set of equations $E^{\prime}=\bar{E} \backslash\left\{e_{i}\right\}$ that is used to solve for the unknown variables $X^{\prime}$. The key steps in the residual generator design is the choice of consistency relation and the solving procedure for the just-determined set of equations.

Structural methods analyze the model structure, i.e., only information about which variables that are included in each equation. This is typically represented by the incidence matrix of a bi-partite graph with node sets $E$ and $X \cup Z$, and each edge in the graph corresponds to a variable dependency in an equation. As an example, the structure of the model in Example 1 can be represented by the incidence matrix

\begin{tabular}{c|cc|cc} 
& $x_{1}$ & $x_{2}$ & $y$ & $u$ \\
\hline$e_{1}$ & $\mathrm{X}$ & $\mathrm{X}$ & & \\
$e_{2}$ & & $\mathrm{X}$ & & $\mathrm{X}$ \\
$e_{3}$ & $\mathrm{X}$ & & $\mathrm{X}$ &
\end{tabular}

For the first step in finding a sequential residual generator, efficient methods from structural analysis [12] exists to, based on the model structure, find all minimal sets of equations with redundancy 1 . Such sets are referred to as a Minimally Structurally Overdetermined (MSO) set of equations, i.e., a set of equations with 1 more equation than variables and where no proper subset of the equations is structurally overdetermined.

In the second step, when a consistency relation has been selected, the square non-linear system of equations $E^{\prime}$ need to be solved. Also here tools from structural analysis is useful. A matching in a bi-partite graph is a subset of edges with no common nodes, i.e., a pairing of equations and variables. From a maximal matching, a computation sequence for the unknown variables $X^{\prime}$ can be obtained.

Definition 1 (Computation sequence). A computation sequence for $M\left(E^{\prime}, X^{\prime}, Z\right)$ is an ordered set $\mathcal{C}=$ $\left\{\left(V_{1}, E_{1}\right), \ldots,\left(V_{n}, E_{n}\right)\right\}$ where $V_{i} \subseteq X^{\prime}$ and $E_{i} \subseteq E^{\prime}$. The order to compute the unknown variables, $X^{\prime}$, in the set of equations, $E^{\prime}$, is defined by the order of appearance in the set.

For example, the computation sequence for the second case in Example 1 is

$$
\mathcal{C}=\left\{\left(\left\{x_{2}\right\},\left\{e_{2}\right\}\right),\left(\left\{x_{1}\right\},\left\{e_{1}\right\}\right)\right\}
$$

indicating that $x_{2}$ is computed from $e_{2}$ and $x_{1}$ is then computed from $e_{1}$. In case of computational loops, i.e., cases where several variables has to be solved for concurrently, sets $E_{i}$ and $V_{i}$ are non-singletons.

\section{B. An Algorithm}

The methodology of investigating all residual generator candidates is used in this paper, and the function FINDRESIDUALGENERATORS that finds and investigates the properties of these in [7] is recalled below. The input variables to the function are the model equations, $E$, the unknown variables, $X$, and an algebraic equation solver, $\mathcal{T} \cdot \mathcal{T}$ solves an equation system, and $\mathcal{T}(\mathcal{C})$ gives the expressions to be used to compute the unknown variables. For example, consider $\mathcal{C}$ in $(2), \mathcal{T}(\mathcal{C})=\left\{x_{2}:=g_{2}(u), x_{1}:=g_{1}\left(x_{2}\right)\right\}$. A sequential residual generator $r_{j} \in R$ consists of a computation sequence and a consistency relation, i.e., $r_{j}=\left(\mathcal{T}(\mathcal{C}), e_{i}\right)$. The call $\operatorname{var}_{X}(\bar{E})$ returns the variables included in $\bar{E}$, the function FINDALLMSOs finds all MSOs given $E$ and $X$, and FINDCOMPUTATIONSEQUENCE investigates the properties of the computation sequence of the unknown variables (see [7] for details). This is done by (1) using the structural model to find a computation sequence, (2) investigate how dynamic equations are used in the computation sequence (see Section II-C for details), and (3) investigate if the algebraic solver $\mathcal{T}$ is able to find expressions for the unknowns variables. If there is no realizable algebraic solution the computation sequence, $\mathcal{C}$, is empty.

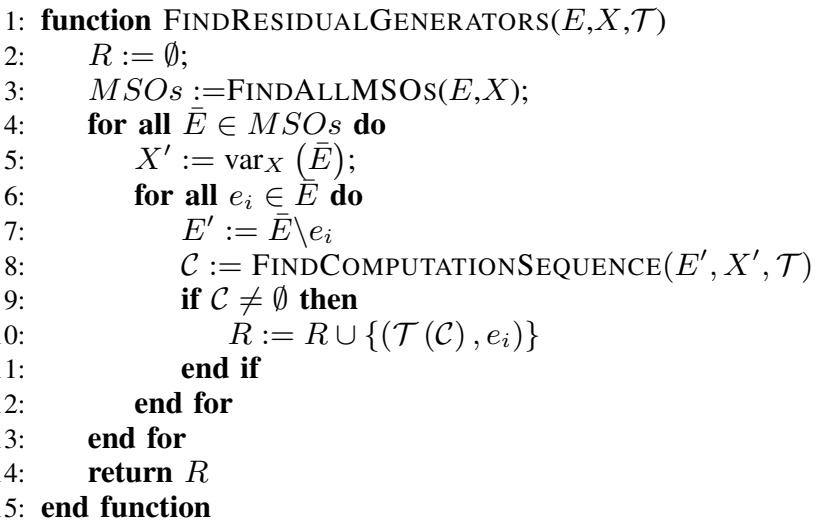




\section{Dynamic Models}

The model used in this investigation is based on equations in the form (1). To make analysis of dynamic models explicit, relations between a variable $x_{i} \in X$ and $\dot{x}_{i}$ is included in the model using an equation

$$
\frac{d}{d t} x_{i}=\dot{x}_{i}
$$

Such an equation can, in a particular computation sequence, be solved in either of two directions [1], [13]

- derivative causality is when $x_{i}$ in (3) is differentiated to obtain $\dot{x}_{i}$, i.e., $\dot{x}_{i}:=\frac{d}{d t} x_{i}$.

- integral causality is when $\dot{x}_{i}$ in (3) is integrated to obtain $x_{i}$, i.e. $x_{i}:=\int \dot{x}_{i} d t+x_{0}$, where $x_{0}$ is the initial value of $x_{i}$.

A computation sequence is said to be in derivative causality if all dynamic constraints are solved in derivative causality and similar for integral causality. If both derivative and integral causality is used in the computations, the sequence is said to be in mixed causality.

\section{Modification to the Algorithm to Handle Dynamic Consis- tency Relations}

The algorithm used to find the properties of the residual generator candidates is described in Section II-B and [7]. To also consider dynamics in the consistency relation, a modification is made to the original algorithm.

An illustrative example is given below to exemplify the case when there is dynamics in the set of equations to be used to construct the residual generator.

$$
\begin{array}{ll}
e_{1}: & \dot{x}_{1}-u=0 \\
e_{2}: & x_{1}-y=0
\end{array}
$$

If $e_{2}$ is selected to be the consistency relation, i.e. $e_{i}=e_{2}$, then $e_{1}$ is used to compute $x_{1}$, and integral causality is used since $\dot{x}_{1}$ is included in $e_{1}$. If, on the other hand, $e_{1}$ is selected as the consistency relation, i.e. $e_{i}=e_{1}$, then $e_{2}$ is used to find $x_{1}$, and the algorithm given in Section II-B gives that neither integral or derivative causality is used since there is no dynamic equation included in the computation sequence (i.e. $e_{2}$ in this case). However, to be able to use $e_{1}$ as the consistency relation, $x_{1}$ has to be differentiated, and to also consider this case and achieve the causalities given in Table I, a slightly modified algorithm is now presented below. There, the call $\operatorname{var}_{D}\left(e_{i}\right)$ returns the differentiated variable, if there is any, in equation $e_{i}$. For example, the call $\operatorname{var}_{D}\left(e_{1}\right)$, where $e_{1}$ is given in (4a), results in $\dot{x}_{1}$. Due to that the model is given in semi-explicit form, a differentiated variable is only included once in the set of equations. This implies that if a differentiated variable is included in the consistency relation, this variable is only known in its undifferentiated form from the computation sequence. Therefore derivative causality is used in such a case. The function IsDerivativePermitted $(\mathcal{D})$ is true if derivative or mixed causality is to be used, otherwise false. The parameter $\mathcal{D}$ is an input parameter and includes information about what causality that is permitted to solve dynamic equations.
TABLE I

THE CAUSALITIES RETURNED FROM THE ALGORITHMS DESCRIBED FOR THE EXAMPLE PRESENTED IN (4)

\begin{tabular}{ccc} 
Consistency relation & Original & Modified \\
\hline$e_{1}$ & Static & Derivative \\
$e_{2}$ & Integral & Integral \\
\hline
\end{tabular}

In FindResidualGenerators all realizable residual generators are saved in $R$, but in the modified algorithm only the realizable residual generators fulfilling the constraint regarding the causality is to be stored in $R$. If $\mathcal{C}$ fulfills the requirements in $\mathcal{D}$ is investigated in the function IsCAUSALITYOK $(\mathcal{C}, \mathcal{D})$.

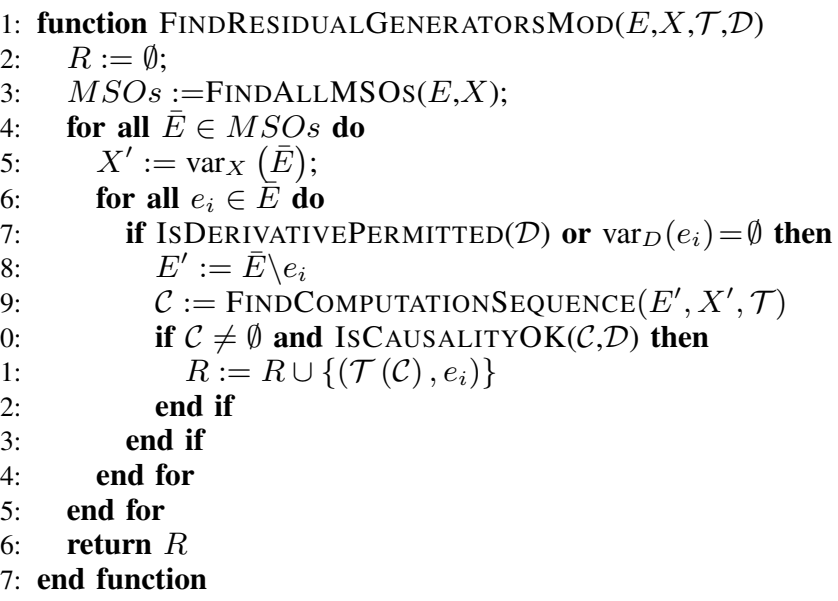

\section{VEHICLE MODEL}

A model of a hybrid electric vehicle (HEV), 32 equations, is used together with sensor models, 5 equations, and fault models, 5 equations. The complete model consisting of the truck model, sensor models and faults are described in Appendix A, Sections III-B and III-C, respectively. For the sake of the analysis to come, relevant parts of the vehicle model in Appendix A are pointed at in Section III-A.

\section{A. Powertrain model}

The modeled vehicle is a long haulage electric parallel hybrid truck, with the connection of the electrical and conventional parts of the powertrain located between the clutch and the gearbox, see Figure 1. The models of the components in the vehicle are based on the model library developed in the Matlab/Simulink based Center for Automotive Propulsion

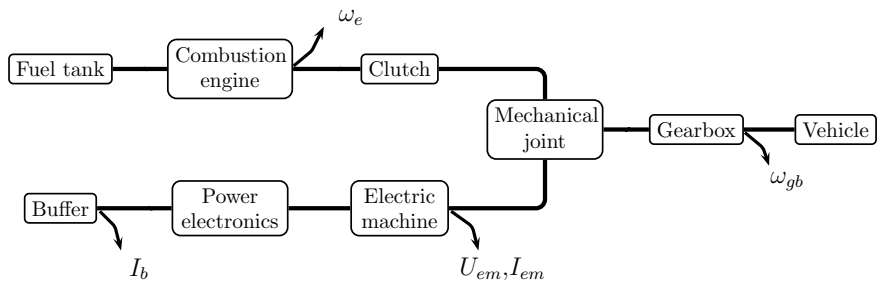

Fig. 1. The configuration of the modeled vehicle. The arrows and signals indicate the sensors used in the diagnosis systems described in Sections IV-B and VI. 
Simulation [14] and the QSS toolbox [15]. Modifications to these models are carried out to model a truck instead of a passenger car, and to include the possibility to induce faults in the models as well as to add sensor noise. The energy management is based on a heuristic control strategy (see [16], [17] for details), and a Willans model is used for the combustion engine [5], [18]. All model equations for the powertrain are given in Appendix A and are described in [16].

1) Battery: The battery is modeled using a Thévenin equivalence circuit, i.e. a voltage source, $U_{o c}$, and an inner resistance, $R_{b}$, connected in series [19]. The battery voltage on the terminals for a current $I_{b}$ is

$$
U_{b}=U_{o c}(S o C)-R_{b} I_{b}
$$

and the state of charge, $S o C$, is based on $I_{b}$ and the capacity of the battery, $Q_{b}$

$$
S o C=S o C_{0}-\frac{1}{Q_{b}} \int I_{b} d t, \quad S o C \in[0,1]
$$

where $S o C_{0}$ is the initial state of charge of the battery.

The modeled battery pack is based on 200 cells. There are two chains of cells connected in parallel, and the cells used are denoted as the "10-Mile PHEV" in [20]. The resistance for the battery pack is assumed to be a constant based on the tabulated resistance at $50 \% \operatorname{SoC}\left(R_{b}=0.023 \Omega\right)$. The total capacity of the battery pack is $33.2 A h$, the $U_{o c}(S o C=0.5)$ is $250 \mathrm{~V}$, leading to an energy storage capacity of approximately $8 \mathrm{kWh}$. The weight of the battery is $150 \mathrm{~kg}$, of this the weight of the cells is $129 \mathrm{~kg}$.

2) Power electronics: The model of the power electronics is assumed to be an ideal component

$$
P_{b}=P_{e m} \Leftrightarrow I_{b} U_{b}=I_{e m} U_{e m}
$$

where $P_{b}$ and $P_{e m}$ are the electrical powers from the battery and motor, and $U_{b}, I_{b}, U_{e m}$, and $I_{e m}$ the battery and electric machine voltages and currents.

3) Electric machine: The electric machine is able to convert electric power to mechanical power and vice verse. A voltage, $U_{e m}$, is applied on the component, resulting in a torque on the outgoing shaft. The torque, $T_{e m}$, is proportional to the armature current, $I_{e m}$, with the torque constant $k_{a}$, except for the frictional losses that are assumed to be proportional to the speed of the machine, $\omega_{e m}$, with the gain $c_{e m, f}$. The speed constant $k_{i}$ is used to model the electromotive force

$$
\begin{aligned}
I_{e m} & =\frac{U_{e m}-k_{i} \omega_{e m}}{R_{e m}} \\
T_{e m} & =I_{e m} k_{a}-c_{e m, f} \omega_{e m}
\end{aligned}
$$

where $R_{e m}$ is the resistance.

In an ideal Permanent Magnet Synchronous Machine (PMSM), that is a common machine type in HEVs due to its high efficiency [21], $k_{i}$ and $k_{a}$ are equal. These constants are defined by $K \phi$, where $K$ is a machine constant that depends on design parameters of the machine, and $\phi$ is the magnetic flux produced by the stator. One way to model the losses of the machine is to use $k_{a} \neq k_{i}$ [5]. This is here done by

$$
k_{a}=k_{i} \eta_{e m, 0}^{\operatorname{sign}\left\{I_{e m}\right\}}
$$

where $\eta_{e m, 0}$ is the efficiency and $\operatorname{sign}\{\cdot\}$ the signum function.

The model is parametrized as a $59 \mathrm{~kW}$ DC machine with constant magnetic flux.

4) Clutch: There is a model of the clutch to handle take off and gear shifts. The clutch model is modified compared to the model presented in e.g. [22], to decrease the stiffness of the simulation model when there is slip in the clutch. The delivered torque from the clutch is given by

$$
T_{c}=u_{c} T_{e}
$$

where $u_{c}$ is the position of the clutch and $T_{e}$ is the delivered torque from the engine. The engine speed is assumed to be

$$
\omega_{e}=\left\{\begin{array}{cc}
\omega_{e, i d l e}, \quad \omega_{m j}<\omega_{e, i d l e} \\
\omega_{m j}, \quad \omega_{m j} \geq \omega_{e, i d l e}
\end{array}\right.
$$

where $\omega_{e}, \omega_{e, i d l e}$, and $\omega_{m j}$ are the engine speed, engine idle speed, and speed in the mechanical joint, respectively.

5) Vehicle: In the vehicle, that has a mass $m_{v}$ and a lumped inertia $J_{\text {tot }}$, the output shaft from the gearbox is connected to the final gear and finally to the wheels. The gear ratio in the final gear is $u_{f}$, and the angular acceleration of the wheels is calculated based on the resulting torque, $T_{n e t}$, acting on the wheels, whose radius is $r_{w}$

$$
\begin{aligned}
T_{n e t} & =T_{g b} u_{f}-T_{d}-T_{r}-T_{b}-T_{g} \\
\dot{\omega}_{w} & =\frac{T_{n e t}}{J_{t o t} u_{f}^{2}+m_{v} r_{w}^{2}}
\end{aligned}
$$

where $T_{g b}, T_{d}, T_{r}, T_{b}$, and $T_{g}$ are the torques from the gearbox, air drag, rolling resistance, mechanical brakes, and slope of the road, respectively.

\section{B. Sensors}

The five sensors available for the diagnosis system used in the analysis of the model in Section IV-B and the simulation study described in Section VI are shown in Figure 1. The engine speed is $\omega_{e}$, and $\omega_{g b}$ is the speed of the outgoing shaft from the gearbox. The naming convention of a sensor signal is the name of the measured variable with the extension SENS in the subscript, e.g. $\omega_{g b, s e n s}$.

The measurement signal, $y$, is given by the noise free signal, $y^{*}$, added with noise, $\tilde{\nu}$

$$
y=y^{*}+\tilde{\nu}
$$

\section{Induced faults}

To model that the battery, power electronics, and the electric machine may break down, two parameter values and two voltages in these models have the possibility to be modified. Note that these faults are only examples of how a fault in these components can be represented in the model. The following modifications of the signals are introduced to model the faults where the nominal signals are denoted by the superscript nom:

$$
\begin{aligned}
f_{b, s c} & : U_{b}=\left(1+f_{b, s c}\right) U_{b}^{n o m} \\
f_{p e} & : U_{e m}=\left(1+f_{p e}\right) U_{e m}^{n o m} \\
f_{e m, \eta}: & \eta_{e m, 0}=\left(1+f_{e m, \eta}\right) \eta_{e m, 0}^{n o m} \\
f_{e m, R}: & R_{e m}=\left(1+f_{e m, R}\right) R_{e m}^{n o m}
\end{aligned}
$$


TABLE II

VALUES OF THE FAULTS INDUCED IN THE MODEL.

\begin{aligned} & Faults Value \\ & \hline$f_{\text {em }, \eta}-0.5 \\ & f_{\text {em, }, R}-0.5 \\ & f_{p e}-0.5 \\ & f_{b, s c}-0.5 \\ & f_{\text {em }, U, \text { sens }} 20 \mathrm{~V} \\ &$\hline\end{aligned}

where $f_{b, s c}$ models that all cells in the battery are not used due to an internal short circuit, $f_{p e}$ is a fault in the power electronics, and $f_{e m, \eta}$ and $f_{e m, R}$ are two faults in the electric machine.

In addition to the four faults modes described in (13a)(13d), the voltage sensor in the electric machine is modeled to possibly include an offset fault, $f_{\text {em, } U \text {,sens }}$

$$
U_{e m, s e n s}=U_{e m}+f_{e m, U, s e n s}
$$

When a fault is induced in the model, the value of the fault is given in Table II.

\section{Selection of Consistency Relation}

Each equation in an overdetermined set of equations can be selected as the consistency relation used to construct a sequential residual generator. However, as described above, the algebraic and dynamic properties of the residual generator may vary with the choice of consistency relation, even though they are based on the same set of model equations. These properties will now be studied. The HEV model in Section III is used, but general conclusions will be made. The presentation is structured so that, first, a simplified but realistic example is used to illustrate the possibility to avoid algebraic loops by consistency relation selection. Secondly, all residual generator candidates based on all MSOs for the vehicle are analyzed regarding algebraic loops and uniqueness, as well as how the causality of the dynamic equations are used. In the latter case, a quantitative analysis shows that the number of possible residual generators differs significantly, thus demonstrating the advantages of the systematic approach.

\section{A. Avoiding algebraic loops by consistency relation selection}

Algebraic loops, as well as multiple solutions of the unknown variables, may occur in computation sequences [1], [23]. There are several numerical and analytical methods available to solve algebraic loops. Linear loops are e.g. easily solved, but non-linear algebraic loops may demand a large computation effort to solve and a solution is not always obtained. Thus, it is sensible to avoid algebraic loops if possible and here the computation sequences are analyzed with respect to algebraic loops.

The possibility to find a unique residual generator given a set of equations in an MSO varies with the chosen consistency relation. To illustrate this an MSO based on the vehicle model presented in Section III-A is used, and since the MSO consists of many equations three simplifications are made in this example:

- the torques due to losses in the vehicle are lumped and denoted $T_{l}$
TABLE III

PERMUTED STRUCTURAL MODEL OF THE SYSTEM GIVEN IN (14) EXCEPT $e_{1}$ THAT IS CHOSEN TO THE CONSISTENCY RELATION. EQUATIONS $e_{2}$ AND $e_{3}$ FORM AN ALGEBRAIC LOOP FOR $I_{e m}$ AND $U_{e m}$.

\begin{tabular}{c|cccccc} 
& $\omega$ & $U_{b}$ & $\dot{\omega}$ & $I_{b}$ & $U_{e m}$ & $I_{e m}$ \\
\hline$e_{7}$ & $\mathrm{X}$ & & & & & \\
$e_{6}$ & & $\mathrm{X}$ & & & & \\
$e_{5}$ & $\mathrm{X}$ & & $\mathrm{X}$ & & & \\
$e_{4}$ & & $\mathrm{X}$ & & $\mathrm{X}$ & & \\
$e_{3}$ & & $\mathrm{X}$ & & $\mathrm{X}$ & $\mathrm{X}$ & $\mathrm{X}$ \\
$e_{2}$ & $\mathrm{X}$ & & & & $\mathrm{X}$ & $\mathrm{X}$
\end{tabular}

- the torques $T_{e}$ and $T_{l}$ are assumed to be known

- the angular speeds in the powertrain are equal and are denoted $\omega$

resulting in the following set of equations

$$
\begin{aligned}
e_{1}: & T_{e}+\underbrace{\eta_{e m, 0}^{\operatorname{sign}\left\{I_{e m}\right\}} k_{i} I_{e m}-c_{e m, f} \omega}_{T_{e m}}-T_{l}(\omega)-J_{t o t} \dot{\omega}=0 \\
e_{2}: & \frac{U_{e m}-\omega k_{i}}{R_{e m}}-I_{e m}=0 \\
e_{3}: & I_{b} U_{b}-I_{e m} U_{e m}=0 \\
e_{4}: & U_{o c}-U_{b}-R_{b} I_{b}=0 \\
e_{5}: & \frac{d}{d t} \omega-\dot{\omega}=0 \\
e_{6}: & U_{b}-y_{1}=0 \\
e_{7}: & \omega-y_{2}=0
\end{aligned}
$$

where $y_{1}$ and $y_{2}$ are sensor signals, and $\omega, \dot{\omega}, U_{b}, I_{b}, U_{e m}$, and $I_{e m}$ are the six unknown variables.

If $e_{1}$ in (14) is selected as the consistency relation, the permuted structural model of the just-determined part, i.e. $\left\{e_{2}-e_{7}\right\}$, is given in Table III. The corresponding computation sequence is

$$
\begin{aligned}
\mathcal{C}=\left\{\left(\{\omega\},\left\{e_{7}\right\}\right),\left(\left\{U_{b}\right\},\left\{e_{6}\right\}\right),\left(\{\dot{\omega}\},\left\{e_{5}\right\}\right),\right. \\
\left.\left(\left\{I_{b}\right\},\left\{e_{4}\right\}\right),\left(\left\{I_{e m}, U_{e m}\right\},\left\{e_{2}, e_{3}\right\}\right)\right\}
\end{aligned}
$$

The pair $\left(\left\{I_{e m}, U_{e m}\right\},\left\{e_{2}, e_{3}\right\}\right)$ indicates that there is an algebraic loop, that also can be seen in Table III. This loop has the non-unique solution

$$
I_{e m}=-\frac{\omega k_{i}}{2 R_{e m}} \pm \sqrt{\left(\frac{\omega k_{i}}{2 R_{e m}}\right)^{2}+\frac{I_{b} U_{b}}{R_{e m}}}
$$

If one of $e_{2}, e_{3}$, or $e_{4}$ is used as consistency relation instead of $e_{1}$, there is no algebraic loop in the just-determined part. The key to avoid the algebraic loop in this example is to find a computation sequence where $I_{e m}$ is computed in $e_{1}$. The computation sequence of the unknown variables if e.g. $e_{2}$ is used as the consistency relation will be

$$
\begin{aligned}
\mathcal{C}=\left\{\left(\{\omega\},\left\{e_{7}\right\}\right),\left(\left\{U_{b}\right\},\left\{e_{6}\right\}\right),\left(\{\dot{\omega}\},\left\{e_{5}\right\}\right),\right. \\
\left.\left(\left\{I_{b}\right\},\left\{e_{4}\right\}\right),\left(\left\{I_{e m}\right\},\left\{e_{1}\right\}\right),\left(\left\{U_{e m}\right\},\left\{e_{3}\right\}\right)\right\}
\end{aligned}
$$

Since the equations in the substitution chain are uniquely solvable, a unique residual generator is found.

Remark 1. Note that since consistency based diagnosis is used, it is possible to construct a test that is based on a 
residual generator with several solutions. As long as at least one of the possible residuals, $r_{1}, r_{2}, \ldots, r_{n}$ where $n$ is the number of solutions, is close to zero, the residual will not react on the fault

$$
\left|r\left(t_{k}\right)\right|=\min \left\{\left|r_{1}\left(t_{k}\right)\right|,\left|r_{2}\left(t_{k}\right)\right|, \ldots,\left|r_{n}\left(t_{k}\right)\right|\right\}, n \geq 2
$$

However, the computation complexity of the system increases if more than one residual are to be evaluated in a diagnostic test.

Remark 2. Sensor equations are often selected to be the consistency relation, but with respect to algebraic loops it is in general preferable to include such an equation in the computation sequence. The reason is that a sensor equation only includes one unknown variable, and therefore can never be part of a loop in the computation sequence. This is exemplified above, where algebraic loops occur if any sensor equation is selected as consistency relation.

1) Series wound electric machine: In the example above the magnetic field, $\phi$, created by the stator or armature in the electric machine is assumed to be constant. This is the case in permanent magnet synchronous machines, which is the machine type mainly used for vehicle propulsion in HEVs. But if a series wound machine is used instead, that e.g. is used in starter motors [24], then $e_{1}$ and $e_{2}$ in (14) are modified to

$$
\begin{aligned}
& e_{1}: \quad T_{e}+\underbrace{\eta_{e m, 0}^{\operatorname{sign}\left\{I_{e m}\right\}} k_{i} I_{e m}^{2}-c_{e m, f} \omega}_{T_{e m}}-T_{l}(\omega)-J_{t o t} \dot{\omega}=0 \\
& e_{2}: \quad \frac{U_{e m}}{R_{e m}+\omega k_{i}}-I_{e m}=0
\end{aligned}
$$

due to that $\phi$ increases linearly with the current in the rotor and stator according to

$$
\phi=k_{a} I_{e m}=\eta_{e m}^{\operatorname{sign}\left\{I_{e m}\right\}} k_{i} I_{e m}
$$

The same variables are included in each equation in (14) and (18), and therefore the structural models are the same for the two machine types. It is however not possible to chose a consistency relation that results in a unique expression for the residual generator for the latter machine type. There are three equations, $e_{1}, e_{2}$, and $e_{3}$, that can be used to find a matching for $I_{e m}$. If $e_{2}$ or $e_{3}$ is used to compute $I_{e m}$ there is a non-linear algebraic loop that has multiple solutions (similar to (16)), and $I_{e m}$ is quadratic in $e_{1}$.

\section{B. Properties of the sequential residual generators candidates}

Now return to the task of finding candidates for sequential residual generators. Given the powertrain model presented in Section III-A and the five sensors available when designing the diagnosis systems (see Figure 1), 99 MSOs are found. For these MSOs there are 2667 sequential residuals generator candidates that are investigated using the algorithm described in Section II-D. The number of these residual generator candidates that fulfill different causality requirements, i.e. mixed, integral, derivative, and static, are presented in Figure 2. In the figure the white bars indicate the number of model equations, i.e. the number of residual generator candidates, included in each MSO. All equations are not invertible and the number of residual generators that are realizable and using corresponding causality are given by the gray bars. The black bars represent how many of the equations that can be selected as a consistency relation to achieve a unique residual generator for each causality. Note that static residual generators are included in Figures 2(a)-2(c), and that pure derivative or integral causality residual generators are included in Figure 2(a).

For MSOs 15-99 there is only a small fraction of the equations that can be used as a consistency relation if only derivative or integral causality is used, and there are 5 MSOs that it is not possible to construct a unique residual generator by using mixed or integral causality (see the non-black bars in Figure 2(a) and 2(b)). However, if the constraint regarding unique residual generators is relaxed to allow multiple solutions, as for example in (16), it is possible to design tests based on all MSOs if integral or mixed causality is used (all MSOs in Figures 2(a) and 2(b) contain black or gray bars).

When derivative causality is used to solve the dynamic equations, it is found that only 43 MSOs can be used to construct diagnosis tests (the black bars in Figure 2(c)). Additional investigations show that this leads to that full fault isolability is not achieved. However, full fault isolability is structurally achieved when mixed or integral causality is used.

Table IV includes the same type of results as Figure 2, but instead gives aggregated numbers. The numbers in parenthesis is the result, when adequate, from the original algorithm FINDRESIDUALGENERATORS described in Section II-B to be compared with the results from FINDRESIDUALGENERATORSMOD described in Section II-D, and it is clear that including the consistency relation in the analysis affects the results. I, D, and $\mathrm{S}$ indicate that integral causality, derivative causality, and static expressions are used.

TABLE IV

THE NUMBER OF MSOS AND SEQUENTIAL RESIDUAL GENERATORS WITH DIFFERENT ALGEBRAIC AND DYNAMIC PROPERTIES. IN TOTAL THERE ARE 2667 RESIDUAL GENERATOR CANDIDATES. COMBINATIONS OF S, D, AND I OCCUR, AND E.G. SI INDICATES THAT BOTH STATIC EXPRESSIONS AND INTEGRAL CAUSALITY MAY BE USED. THE FIGURES IN PARENTHESIS IS THE RESULT, WHEN ADEQUATE, FROM THE ORIGINAL ALGORITHM DESCRIBED IN SECTION II-B.

\begin{tabular}{c|c|cc} 
Algebraic constraint & Causality & \# MSO sets & \# res. gen. \\
\hline & SDI & 94 & 1374 \\
Unique & SI & $94(94)$ & $334(414)$ \\
residuals & SD & 43 & 70 \\
& S & $6(6)$ & $33(33)$ \\
\hline & SDI & 99 & 1520 \\
Realizable & SI & $99(99)$ & $373(458)$ \\
residuals & SD & 43 & 70 \\
& S & $6(6)$ & $33(33)$ \\
\hline
\end{tabular}

\section{Summary and discussion}

In the illustrative example in Section IV-A it was shown that the occurrence of algebraic loops varies with the selection of consistency relation. It was also shown that the possibility to find a unique residual generator depends on the model the residual generator is based on, where in this specific case it is possible to find a unique residual generator using a permanent 


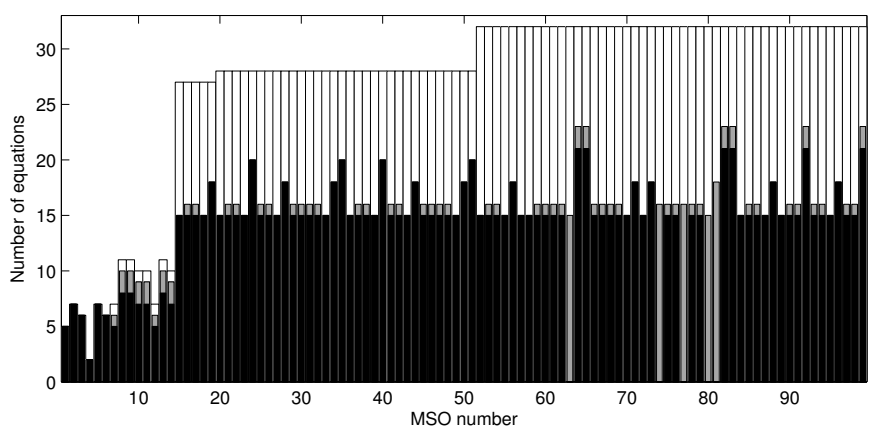

(a) Mixed causality.

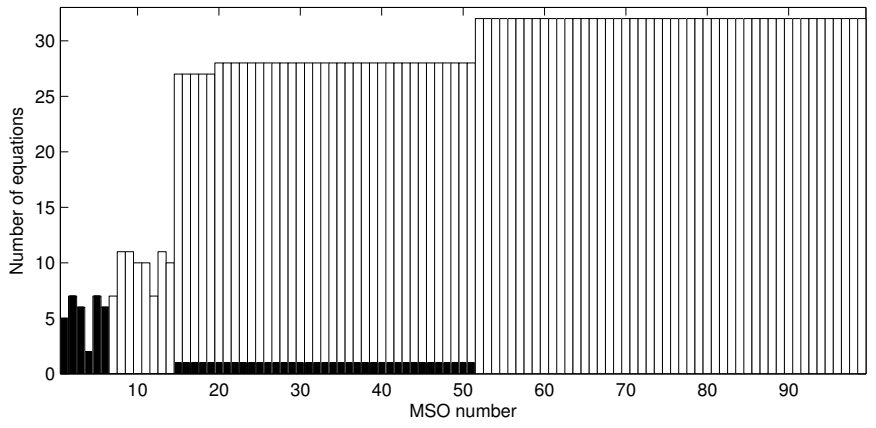

(c) Derivative causality.

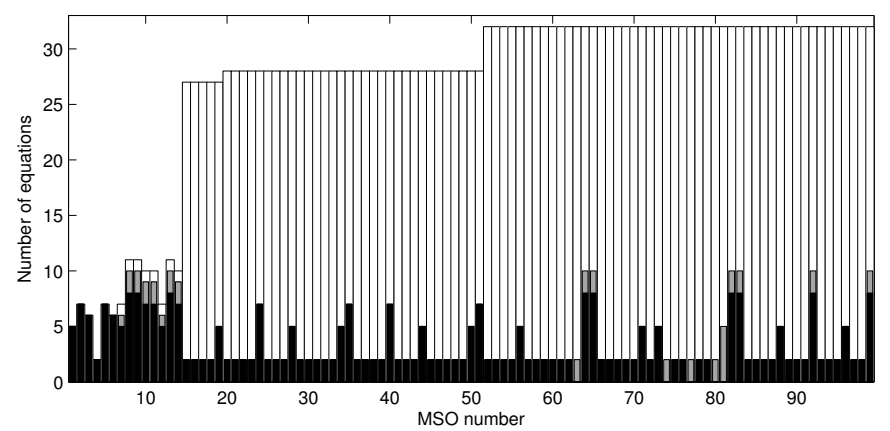

(b) Integral causality.

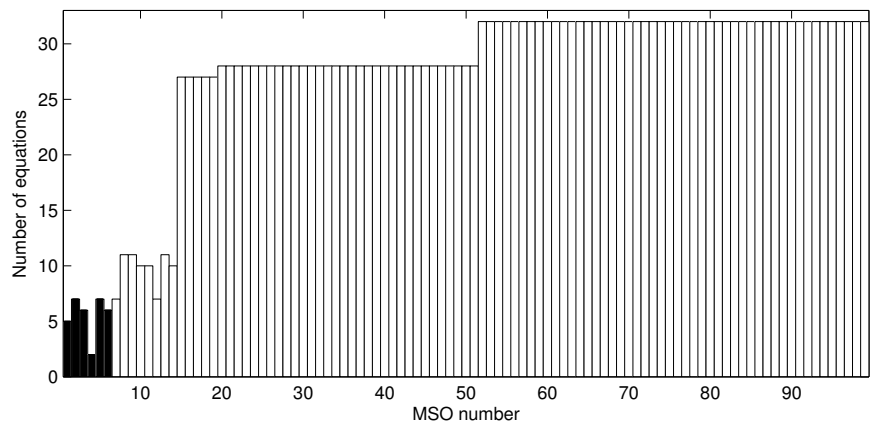

(d) Static residuals.

Fig. 2. The white bars indicate the number of equations in each MSO in the diagnosis systems, the black bars the number of equations that can be selected to consistency relations to fulfill the causality constraint and that a unique sequential residual generator is to be found, and the gray bars if the constraint regarding unique residual generators is relaxed to that a residual generator is to be found (i.e. unique or multiple solutions of the unknown variables).

magnet electric machine, but not if a series wound electric machine is used.

For selection of sequential residual generators, the main result so far is Figure 2 and Table IV. The analysis of the entire vehicle model gives that there is a small fraction of the residual generators that uses integral and derivative causality (compare the black and white bars in Figures 2(b) and 2(c)). In general there are few choices of consistency relations when designing a sequential residual from an MSO to achieve certain properties regarding algebraic solutions and causality. Therefore it can be stated that systematic methods are valuable to investigate the properties of the sequential residual generator candidates.

\section{Methods for Utilization of Residual Generators AND TEST QuANTITIES}

There are several important issues when using the residual generators and test quantities in a diagnosis system, and in this section three particular topics are discussed. First a technique is presented to avoid differentiation in the consistency relation, secondly reinitialization of dynamic residual generators, and finally a way to increase test performance by taking fault sensitivity into consideration. The residual generators are constructed using the algorithm given in Section II-D, and a general model given as a semi-explicit DAE is considered.

\section{A. Avoid differentiating in the consistency relation using a state transformation}

There are several ways of numerically differentiating a signal, see e.g. [25], [26]. However, here a method is de- scribed that leads to that no differentiated variable needs to be computed in the redundant equation.

Since the model is given as a semi-explicit DAE, see (1), the differentiated variable is included linearly in the consistency relation. This leads to a residual generator that can be expressed in the general form

$$
\tilde{r}=\dot{x}_{i}+b(z)
$$

where $x_{i}$ is the variable that occurs in the consistency relation in its differentiated form and $b(z)$ an arbitrary function of known signals. Residuals including differentiated variables are often filtered, and by filtering $\tilde{r}$ in (20a) the residual $r$ is obtained

$$
r=\frac{\alpha}{p+\alpha} \tilde{r}=\frac{\alpha}{p+\alpha}\left(\dot{x}_{i}+b(z)\right)
$$

where $p$ is the differentiation operator and $\alpha>0$ for stability.

Now, it is possible to compute $r$ in (20b), without calculating a differentiated signal using a transformation and state the residual generator in state-space form. The conditions for this to be possible is that $b$ a function of known variables, and the residual is filtered as in (20b) [27]. Using the state

$$
\Gamma=r-\alpha x_{i}
$$

we obtain that the residual generator in (20b) can be expressed as

$$
\begin{aligned}
\dot{\Gamma} & =-\alpha \Gamma-\alpha^{2} x_{i}+\alpha b \\
r & =\Gamma+\alpha x_{i}
\end{aligned}
$$

which is a computational form that does not include a differentiation of variable $x_{i}$. By using this methodology it is possible 
to always avoid differentiating a variable in a sequential residual generator if a signal only is to be differentiated in the consistency relation.

Drift in the state, that is a common issue when using integral causality, is not an issue using this methodology since the filter is asymptotically stable. This is the reason that this residual generator is not considered as using integral causality, even though a signal is integrated in the residual generator.

Remark 3. Another possibility to avoid differentiating a variable to be used in the consistency relation, is by integrating the consistency relation. The residual instead of (20a) would e.g. be

$$
\bar{r}(t)=\int_{t_{0}}^{t} \tilde{r}(\tau) d \tau+r\left(t_{0}\right)=a x(t)+\int_{t_{0}}^{t} b(z(\tau)) d \tau+b\left(z\left(t_{0}\right)\right)
$$

where $b\left(z\left(t_{0}\right)\right)$ is the initial value of $b(z)$. In this case the residual generator has the properties of integral causality, i.e. the initial value of the state has to be available and drift may occur in the integrators due to modeling errors.

Note that it is only differentiated variables in the consistency relation, and not in other parts of the MSO, that always can be avoided to compute by integration. To illustrate, consider the example MSO

$$
\begin{array}{ll}
e_{1}: & x_{2}-u_{1}=0 \\
e_{2}: & \dot{x}_{2}-x_{1}=0 \\
e_{3}: & \sin \left(x_{1}\right)-y_{1}=0
\end{array}
$$

Assume that integral causality is to be used in the residual generator, and that $e_{3}$ is used as the consistency relation. It is not possible to find a sequential residual generator that fulfills this requirement, since $\int x_{1} d t$ is computed from $e_{2}$ in order to not differentiate $x_{2}$, but $x_{1}$ needs to be known in the consistency relation.

\section{B. Initialization of states}

When a dynamic residual generator is started or re-started, the states $\omega \in \mathbb{R}^{n}$ needs to be initialized. It is assumed that the residual generator is given in state space form

$$
\begin{aligned}
\dot{\omega} & =f(\omega, z) \\
r & =h(\omega, z)
\end{aligned}
$$

where $z$ is a vector of known variables. When the states $\omega$ are initialized the residual and its derivatives should ideally be zero in the fault free case. This leads to that the initial states $\omega\left(t_{0}\right)$ should be chosen such that

$$
r\left(t_{0}\right)=\dot{r}\left(t_{0}\right)=\ldots=r\left(t_{0}\right)^{(n-1)}=0
$$

where $t_{0}$ is the time the residual is started.

The state in the first order system given in (20d) and (20e) is initialized using (24) as

$$
\Gamma\left(t_{0}\right)=r\left(t_{0}\right)-\alpha x_{i}\left(t_{0}\right)=-\alpha x_{i}\left(t_{0}\right)
$$

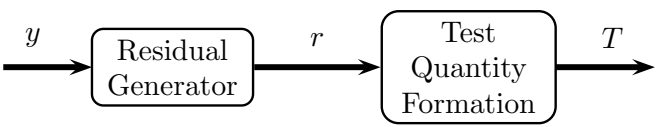

Fig. 3. The residuals are computed from residual generators based on known signals $y$. The residuals are post processed to form test quantities, $T$, that are compared with thresholds to state if the tests have reacted.

\section{Consider fault excitation when computing test quantities}

To increase robustness of decision, residuals are often post processed by computing to what is here referred to test quantities, $T$, see Figure 3. A well known algorithm is CUSUM [4], [28], that in this specific case can be stated as

$$
T\left(t_{k}\right)=\max \left\{0, T\left(t_{k-1}\right)+\left|r\left(t_{k}\right)\right|-\nu\right\}
$$

where $\nu$ is a design parameter that corresponds to the noise and model uncertainty in the residuals. The test reacts when $T$ is above a threshold $J$.

The sensitivity of the residuals often varies with the operating condition of the system to be monitored. One way to improve the performance of the diagnosis system is to consider this aspect when computing the test quantities. A small academic example is used to illustrate the basic principle, and in Section VI-D the approach is applied to the HEV model described in Section III. Consider the system

$$
\begin{aligned}
& x=u \\
& y=(1+f) x
\end{aligned}
$$

where $u$ and $y$ are known signals, $x$ an unknown signal, and $f$ a fault. One residual generator of (27) is

$$
r=y-u
$$

and the internal form, i.e. how the faults affect the residual, of the residual generator is

$$
r=u+f \cdot u-u=f \cdot u
$$

From the internal form it is obvious that the residual sensitivity on the fault depends on the value of $u$.

To achieve good performance in the diagnosis system it is beneficial to only update $T$, as in (26), when the residual is excited by the fault. Figure 4 exemplifies this based on (27)(29) where

$$
u(t)=10 \sin (t)
$$

and $f$ is a constant. In the figure it can be seen that the test does not react on the fault if $T$ is updated also when there is low excitation in the residual, i.e. when $r$ is close to zero even though there is a fault in the system. However, if $T$ only is updated when the magnitude of $y$, that is assumed to be a good approximation of $u$, is above a threshold the fault is detected.

The offset parameter $\nu$ is here set to a fixed value. Instead of not updating $T$ when there is low excitation in the residual, there is a possibility to modify $\nu$. The disadvantage of this is that e.g. when $x=0, \nu>0$ in order to avoid false alarm due to sensor noise, leading to that $T$ is decreasing for this operating point. 

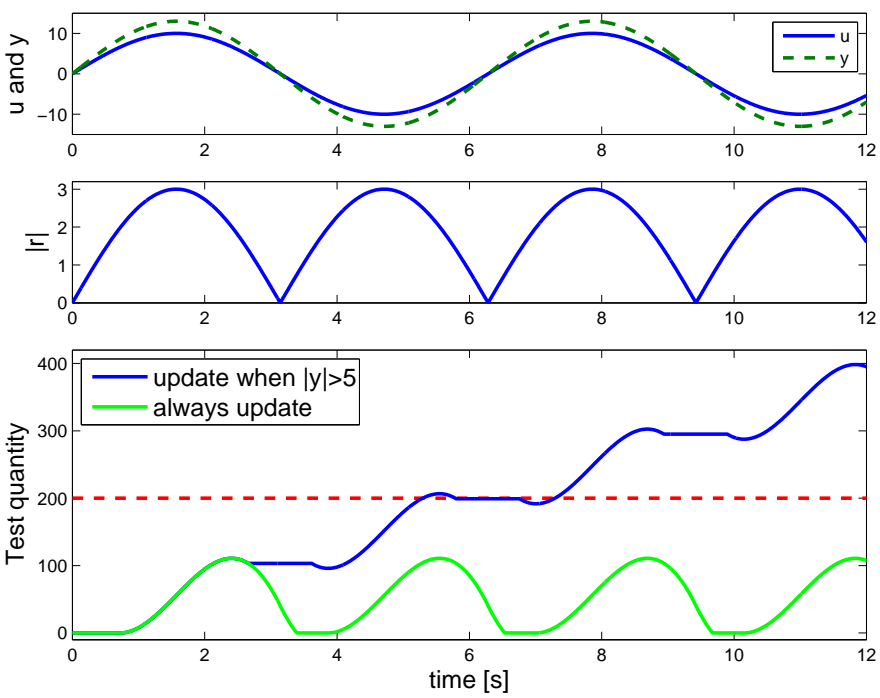

Fig. 4. The variable $u(t)$ is a sinus function with amplitude 10 and $f=0.3$. For the selected $\nu$ and $J=200$ the diagnostic test does not react on the fault if the test quantity, $T$, is updated all the time. However, if $T$ only is updated when $|y|>6$, the test reacts on the fault.

The conclusion from this simple example is that it is advantageous to use fault models to find the internal form of the residual generator, and to use this internal form to design the strategy for not updating the test quantity when there is low excitation in the system.

\section{Illustrative Designs and Simulation Study}

Two diagnosis systems, one based on mixed causality and one on integral causality, of the HEV described in Section III are evaluated to investigate the impact of different choices in the design of a diagnosis system. The general methodologies described in Section V, e.g. initialization of the states and the internal form of the residual generators, are utilized.

\section{A. Properties of diagnosis systems used in simulation study}

The two diagnosis systems use information from five sensors, see Figure 1, and are found using the algorithm FindRESIDUALGENERATORSMOD from Section II-D. If the original algorithm FINDRESIDUALGENERATORS were used, both these two systems would be classified to use integral causality, since the differentiation occurs in the consistency relation itself. Thereby these two systems illustrate the difference between the algorithms as discussed in Section II-D.

1) MCDS: The diagnosis system based on mixed causality is denoted MIXED CAUSALITY DIAGNOSIS SYSTEM, or MCDS for short, and the equations in the computation sequences are uniquely solvable in the residual generators. The diagnosis system consists of four tests that achieves full structural single fault isolability [29] of the five faults. Each test is based on an MSO that is used to construct a sequential residual generator, that are given in Appendix B in (47), (48), (51), and (52). Test 1 is static, Test 2 is based on integral causality, and Tests 3 and 4 are based on mixed causality. In the mixed causality tests the mass of consumed fuel, $m_{f}$, is solved by

$$
m_{f}(t)=\int_{t_{0}}^{t} \dot{m}_{f}(\tau) d \tau+m_{f}\left(t_{0}\right)
$$

Equation (11) is used as consistency relation, but the different torques are computed based on different sensors in the two tests. In the consistency relation $w_{g b}$ is differentiated to compute $\dot{\omega}_{g b}$, resulting in that derivative causality is used.

For the computation of the residual, the reformulation in (20) is used and the residual is expressed in the form

$$
\tilde{r}=\dot{\omega}_{g b}+b
$$

The residual generators are filtered and transformed according to $(20 b)-(20 c)$, that results in

$$
\begin{aligned}
\dot{\Gamma} & =-\alpha \Gamma-\alpha^{2} \omega_{g b}+\alpha b \\
r & =\Gamma+\alpha \omega_{g b}
\end{aligned}
$$

The algebraic loop for $I_{e m}$ and $U_{e m}$ considered in Section IV-A is not an issue in these two residual generators, since $U_{e m}$ is known without using any of $e_{2}$ and $e_{3}$ in (14). The required voltage from the power electronics is known in Test 3, and the sensor measuring $U_{e m}$ is available in Test 4 .

2) ICDS: A diagnosis system based on integral causality and unique expressions of the unknown variables in the computation sequences is designed, and this system is denoted INTEGRAL CAUSALITY DIAGNOSIS SYSTEM, or ICDS for short. It is possible to use the same sets of equations that are used in MCDS, and using the same MSOs the structural isolability properties are the same. Tests 1 and 2 in MCDS can also be used in ICDS, see (47) and (48) for corresponding residual generators, while different consistency relations are to be selected in Tests 3 and 4 in the ICDS since the consistency relations selected in MCDS result in mixed causality.

Tests 3 and 4 are based on MSOs with 29 and 32 equations respectively, but only two of these, $e 32$ and $e 37$ in Appendix A, are possible to select as consistency relations in this system

$$
\begin{array}{ll}
e_{32}: & \omega_{w}=\frac{\omega_{g b}}{u_{f}} \\
e_{37}: & \omega_{g b}=\omega_{g b, \text { sens }}
\end{array}
$$

When using any of these as a consistency relation, $\dot{\omega}_{w}$ is calculated using (11), and $\omega_{w}$ by integrating this signal

$$
\omega_{w}(t)=\int_{t_{0}}^{t} \dot{\omega}_{w}(\tau) d \tau+\omega_{w}\left(t_{0}\right)
$$

In the residual generators used in both Tests 3 and 4, (33) is used as the consistency relation

$$
r=\omega_{g b, s e n s}-\omega_{w} u_{f}
$$

and the residual generators are presented in (49) and (50). Note that the only difference between these residual generators and the ones used for Tests 3 and 4 in MCDS are the last four equations in the computation sequences and the consistency relations. In the residual generators in MCDS the consistency relation is $e_{30}$ and the last part of the computation sequence 
$\left(\left\{\omega_{w}\right\},\left\{e_{32}\right\}\right),\left(\left\{T_{d}\right\},\left\{e_{25}\right\}\right),\left(\left\{T_{r}\right\},\left\{e_{26}\right\}\right),\left(\left\{T_{n e t}\right\},\left\{e_{29}\right\}\right)$, while $e_{32}$ is used as the consistency relation in Tests 3 and 4 in ICDS and there is a dynamic loop in the computation sequence $\left(\left\{T_{d}, T_{r}, T_{n e t}, \omega_{w}\right\},\left\{e_{25}, e_{26}, e_{29}, e_{30}\right\}\right)$.

Due to that it is only Tests 3 and 4 that are different in MCDS and ICDS, only these two tests are considered in the simulation study.

\section{B. Model used in the diagnosis system}

The model of the vehicle powertrain used in the diagnosis systems is the same as the model presented in Section III-A and in Appendix A, except for the clutch model. To investigate the consequences of not having a valid model in all operating modes of the system to be monitored, it is assumed that the model of the clutch only is available when the clutch is fully engaged. This results in that when the clutch is disengaged or there is slip in the clutch, corresponding test quantities are not updated and no faults are to be detected in these tests.

\section{Initialization of states when restarting residual generators}

The time it takes for a transient in a dynamic residual generator to fade out after it is initialized decreases if the states in the residual are accurately initialized. When the model is not valid in all operating points it is therefore more important to accurately initialize the states since the residual is restarted when the model becomes valid. The basic idea when initializing the states is to use (24). There are several possibilities to reduce the sensor noise impact on the initialization of the state. In e.g. [30] this is done by assuming Gaussian noise and finding the initial value of the state using a least square estimate over a time window. A less complex method is to filter the signal to be used in the initialization using a time constant, $\tau$. This method is used here, and the state $w_{w}$ calculated from (35) and (11) in ICDS is reinitialized in the residual generators when the vehicle model used in the diagnostic tests is becoming valid using $r\left(t_{0}\right)=0$ in (36)

$$
\omega_{w}\left(t_{0}\right)=\left.\frac{1}{\tau_{w} p+1} \frac{\omega_{g b, s e n s}(t)}{u_{f}}\right|_{t=t_{0}}
$$

The fuel consumed, $m_{f}$, is a state in addition to $\omega_{w}$ in Test 3 , while there are two additional states in Test $4 ; m_{f}$ and $S o C$. The states except $\omega_{w}$ have slow dynamics and are therefore not reinitialized when the diagnostic model becomes valid. Instead the states are only updated when the model is valid.

The state used in the transformation in MCDS is reinitialized when the model used in the diagnosis system is getting valid by using (25), where $x_{i}=\omega_{g b}$ according to (32). It is assumed that the powertrain is fault free in the initialization of $\Gamma$, i.e. $r\left(t_{0}\right)=0$, and $\omega_{g b, s e n s}$ is used instead of $\omega_{g b}$

$$
\Gamma\left(t_{0}\right)=-\left.\frac{1}{\tau_{\Gamma} p+1} \alpha \omega_{g b, s e n s}(t)\right|_{t=t_{0}}
$$

\section{Two approaches for when to update dynamic test quantities}

As stated above, it is assumed that the monitored system is fault free and the residual is zero in the initialization of the states in both MCDS and ICDS. If the equations used in the expression for the signal to be integrated are inconsistent with the monitored system, the integrated signal will drift from the true value. To increase the fault sensitivity of corresponding test quantities, it is preferable to only update the test quantities when the residuals are non-zero even though the estimation of the signal to be integrated is inconsistent (see Figure 4). Two approaches to find updating conditions for the test quantities are presented below.

1) Fixed time: The first approach is to not update the dynamic test quantities in the diagnosis system before a time, $t_{d}$, after a test has been valid in order for the fault to have time to affect $r$. This means that the test quantity is updated when

$$
t>t_{0}+t_{d}
$$

where $t_{0}$ is the time of the latest reinitialization of the states.

2) Internal form: The second approach requires fault models that are used to investigate how the faults affect the residuals by finding the internal form of the residual generators, see Section V-C. This approach is implemented and compared with the fixed time approach given above using Test 4 in ICDS. The test is expected to react on $f_{e m, \eta}, f_{b, s c}$, and $f_{e m, U, s e n s}$, but for simplicity only $f_{e m, \eta}$ and $f_{e m, U, s e n s}$ are considered here. These faults lead to different internal forms of the residual generator and therefore several test quantities are constructed that use different updating conditions.

First, the internal form of Test 4 in ICDS when there is a fault in the voltage sensor in the electric machine is found by the substitution chain given by the computation sequence (50)

$$
r(t)=\frac{f_{\text {em }, U, \text { sens }}}{f_{\text {em }, U, \text { sens }}+1} \cdot C \int_{t_{0}}^{t} u_{g b} I_{e m}(\tau) \eta_{e m, 0}^{\operatorname{sign}\left\{I_{e m}(\tau)\right\}} d \tau
$$

where $C$ is a constant and $u_{g b}$ the gear ratio in the gearbox. Due to (40) it is obvious that the fault excitation is dependent on the magnitude of the integral. This leads to that a condition for when to update the test quantity to achieve good fault detection performance is

$$
\left|\int_{t_{0}}^{t} u_{g b}(\tau) I_{e m}(\tau) \eta_{e m, 0}^{\operatorname{sign}\left\{I_{e m}(\tau)\right\}} d \tau\right|>J_{1}
$$

where $J_{1}$ is a design parameter. A comparison of the conditions in (39) and (41) is shown in Figure 5. As expected, the test quantity that is updated using (41) does not decrease in the second time interval the model is valid and there is low fault excitation in the residual. Note that there is low fault excitation in the residual at this time interval even though the electric machine is used. This is due to that the machine frequently switches from generator to motor mode, see Figure 5.

The internal form of the residual generator for $f_{e m, \eta}$

$$
\begin{aligned}
& r(t)=C \int_{t_{0}}^{t} u_{g b}(\tau) I_{e m}(\tau) \eta_{e m, 0}^{\operatorname{sign}\left\{I_{e m}(\tau)\right\}} . \\
& \cdot\left(\left(1+f_{e m, \eta}\right)^{\operatorname{sign}\left\{I_{e m}(\tau)\right\}}-1\right) d \tau
\end{aligned}
$$

Finding the times $\tau \in\left\{t_{0}, t\right\}$ when $I_{e m}(t) \geq 0 A$ and $I_{e m}(t)<$ $0 A$ 

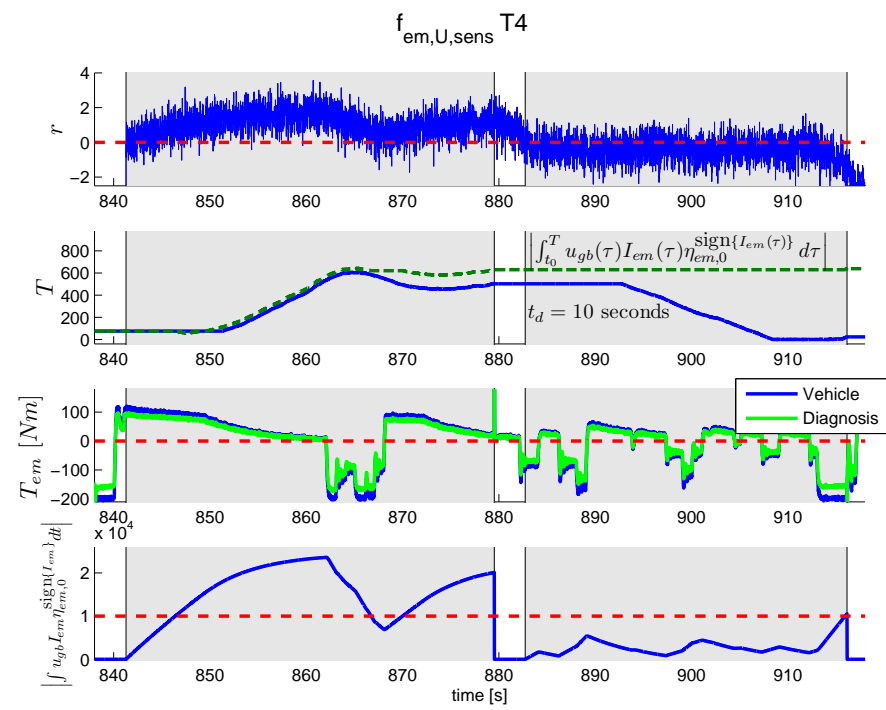

Fig. 5. The residual and test quantities for Test 4 in ICDS when $f_{\text {em, } U \text {,sens }}$ has occurred, and the shaded areas indicate when the model used in the diagnosis system is valid. Two different conditions, (39) and (41), for when the test quantity is to be updated are compared, using $t_{d}=10 \mathrm{~s}$ and $J_{1}=10000 A s$. The torque $T_{e m}$ is almost proportional to $I_{e m}$, see (8b), and the operating points of the electric machine thereby affect how long time the test quantity is updated when (41) is used as the update condition.

$$
\begin{aligned}
& t^{+}=\left\{\tau \in\left\{t_{0}, t\right\}: I_{e m}(t) \geq 0 A\right\} \\
& t^{-}=\left\{\tau \in\left\{t_{0}, t\right\}: I_{e m}(t)<0 A\right\}
\end{aligned}
$$

leads to that (42) can be written as

$$
\begin{aligned}
r(t)=f_{e m, \eta} \cdot & C \int_{t^{+}} u_{g b} I_{e m}(\tau) \eta_{e m, 0} d \tau+ \\
& +\frac{f_{e m, \eta}}{1+f_{e m, \eta}} \cdot C \int_{t^{-}}(-) u_{g b} \frac{I_{e m}(\tau)}{\eta_{e m, 0}} d \tau
\end{aligned}
$$

Under the condition that $\left.\left.f_{e m, \eta} \in\right]-1,0\right]$, which is a reasonable assumption according to (8c) and (13c), $\dot{r}<0$ for both positive and negative $I_{e m}$. This leads to the following condition for when to update the test quantity

$$
\int_{t_{0}}^{t} u_{g b}\left|I_{e m}(\tau) \eta_{e m, 0}^{\operatorname{sign}\left\{I_{e m}(\tau)\right\}}\right| d \tau>J_{2}
$$

where $J_{2}$ is a design parameter. The difference between (41) and (45) is that the latter test quantity is updated as long as the electric machine is used, even though it changes operation mode between generator and motor. Figure 6 presents the test quantities achieved using the updating conditions in (39), (41), and (45) when $f_{e m, \eta}$ has occurred. It can be seen that the test quantity updated when (45) is true reacts better than the test quantity updated when (41) is used, since the first is updated in both time intervals the model is valid and the specific fault excite the residual at these times.

Remark 4 (Several test quantities based on the same residual). Based on Figures 5 and 6, two different conditions are derived for when to update the test quantity, i.e. (41) and (45), in order for Test 4 to react on both $f_{\text {em, } U \text {,sens }}$ and $f_{\text {em, } \eta}$ in a good way. Thus, it makes sense to compute several test quantities based on the same residual. The drawback is the increase in computational complexity.

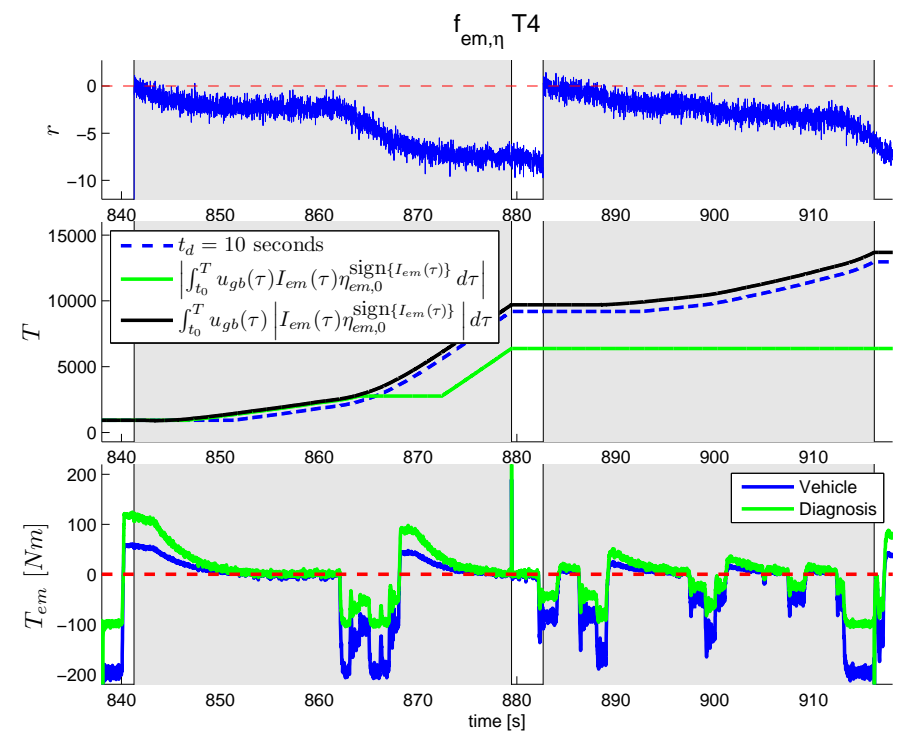

Fig. 6. Test quantities for Test 4 in ICDS based on the same residual but different updating conditions, when $f_{e m, \eta}$ has occurred. The test quantities are updated with the conditions given by (39), (41) and (45). In contradiction to when $f_{\text {em, } U \text {, sens }}$ has occurred (see Figure 5), $\dot{r}<0$ both when the machine operates as motor and generator, as indicated in (44).

\section{E. Simulations on driving cycle}

A simulation study is carried out to evaluate the designed diagnosis systems. The faults described in (13) are induced in the vehicle model one by one. The driving cycle used is FTP75 and the speed profile can be found in e.g. [5].

1) Normalized test quantities: Test quantities are computed using the CUSUM algorithm presented in Section V-C. To compare the performance of different diagnosis systems, normalized test quantities, $T_{\text {norm }}$, are calculated based on the maximum value, $T_{\max , \mathrm{NF}}$, of $T$ in the fault free case when FTP75 is used. An alarm is generated when $T_{\text {norm }}>1$ and the design parameter $\Phi \in[0,1]$ states the margin to false alarm for the specific driving cycle.

$$
T_{\text {norm }}=\frac{T}{T_{\max , \mathrm{NF}}} \Phi
$$

2) Residual responses: Residuals for the diagnosis systems are compared, and in Figure 7 the residuals used in Tests 3 in MCDS and ICDS are shown for the fault free case as well as when $f_{e m, \eta}$ has occurred. The residuals are normalized so the standard deviation of the residuals in the fault free case is one. The residual used in ICDS is more affected by the fault compared to the residual in MCDS, and this is the general result in the simulation study when the different faults are induced in the vehicle model.

The method used to reinitialize the states in the residuals when the model is getting valid is working properly, since the values of the residuals are close to zero after the initialization.

3) Test quantity responses: The residuals are post processed as described above, and all faults are isolated in both MCDS and ICDS. The test quantities generally react stronger in ICDS even though the tests in the two systems are based on the same sets of model equations. In Figure 8 the test 

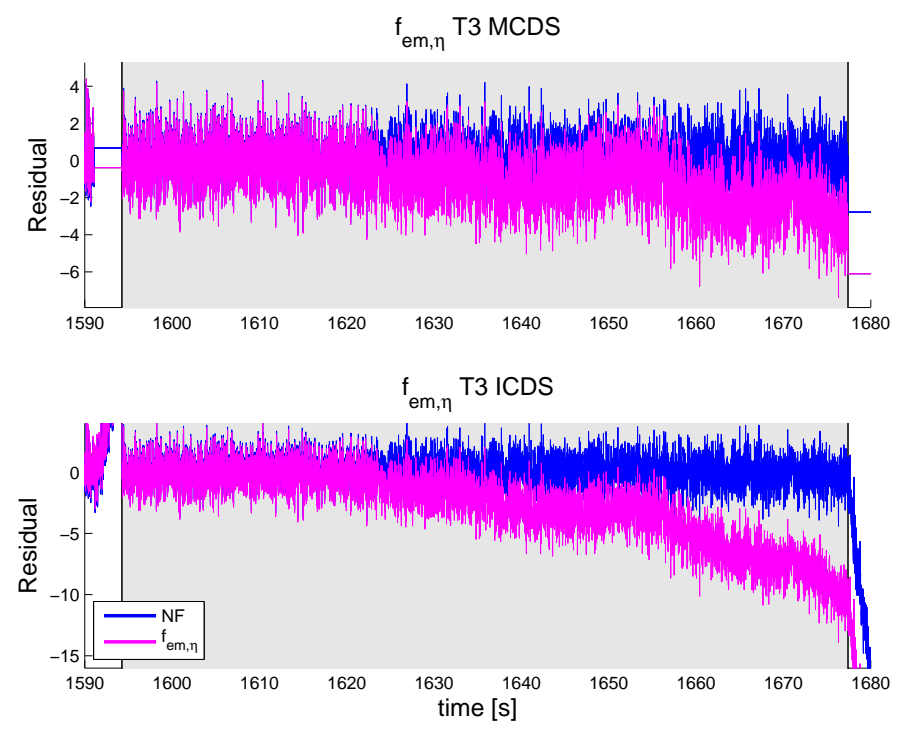

Fig. 7. The residual used in Test 3 in ICDS is more affected to $f_{e m, \eta}$ compared to corresponding residual in MCDS. The residuals are normalized so the standard deviation in the fault free case is one, and the shaded areas indicate when the model used in the diagnosis system is valid, i.e. from 1594 seconds to 1677 seconds.

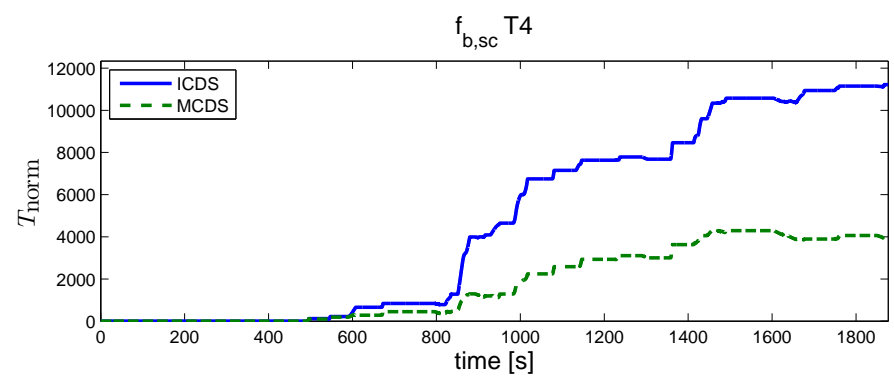

Fig. 8. Normalized test quantities for Test 4 in MCDS and ICDS when there is a short circuit in the battery after 400 seconds. The test quantities are normalized according to (46) with $\Phi=0.5$, and $T_{\text {norm }}$ using integral causality is larger than $T_{\text {norm }}$ using mixed causality after the fault has occurred.

quantities are shown when there is a short circuit in the battery after 400 seconds.

The different updating conditions for the test quantities are implemented in ICDS to evaluate the impact of these different design selections using the entire driving cycle. As indicated in Figures 5 and 6, the tests generally react stronger on the fault if the internal form of the residual is considered when designing test quantities. In Figure 9 this is exemplified when $f_{e m, U, s e n s}$ has occurred and the test quantity that is updated using the condition based on the internal form for the specific fault, i.e. (41), reacts better than the other test quantities.

\section{F. Summing up}

The method used to initialize the states in the diagnosis systems is straight forward and is working properly, i.e. the residuals are close to zero after the initialization. The use of the internal form is demonstrated to be advantageous, according to (41), (45) and Figures 5, 6, and 9. Note that in order for Test 4 to react on both $f_{e m, U, s e n s}$ and $f_{e m, \eta}$ in a good way, two test quantities based on the same residual using

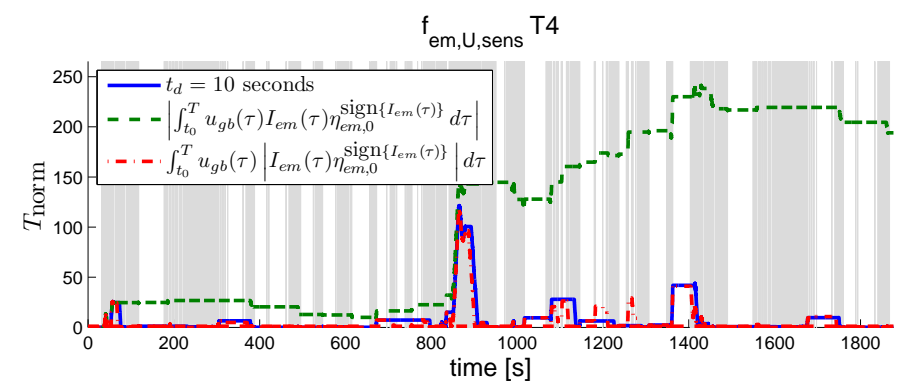

Fig. 9. The test quantities for Test 4 in ICDS using different conditions for when to update the test quantities and there is a fault in the voltage sensor in the electric machine at $t=0 \mathrm{~s}$. The shaded areas indicate when the model used in the diagnosis system is valid. At $t=840 \mathrm{~s}$ the system is excited and the diagnostic tests react on the fault (see Figure 5). Note that it is only the test quantity that is updated using the internal form of the fault, i.e. (41), that reacts from this time till the end of the driving cycle.

different updating conditions are used, since the faults affect the residual in different ways. Since the uncertainties in the study predominantly relate to disturbances rather than model inaccuracies, then it is reasonable that ICDS performs better than MCDS. Therefore it is valuable to use the modified algorithm FINDRESIDUALGENERATORSMOD that singles out such systems.

\section{CONCLUSIONS}

A reduced HEV model was used to illustrate how the selection of consistency relation affects the occurrence of unique expressions of the unknowns and algebraic loops in sequential residual generators. All sequential residual generator candidates of the vehicle model were investigated with respect to algebraic and dynamic properties, and it was found that in general there are few selections of consistency relations in an MSO that achieves predefined properties. Thereby it can be stated that systematic methods analyzing the properties of the residual generator candidates are valuable.

Having selected the residuals, important aspects remain when utilizing them in a diagnosis system. A straight forward method, given in (24) and e.g. (37), to reinitialize the states in the residual generators was shown to work properly. It was also shown that the method to update the test quantities based on the internal form of the residual generators, significantly increased the diagnosis performance. Simulations verified that it is beneficial to use several test quantities based on the same residual using different updating conditions when a residual is sensitive for several faults.

All in all, it has been shown that the engineering support the used methods gives was a key to design well behaved diagnosis systems. The methods are general in character and provides a useful methodology when designing diagnosis systems for HEVs or other complex systems. 


\section{APPENDIX}

\section{A. Powertrain Model}

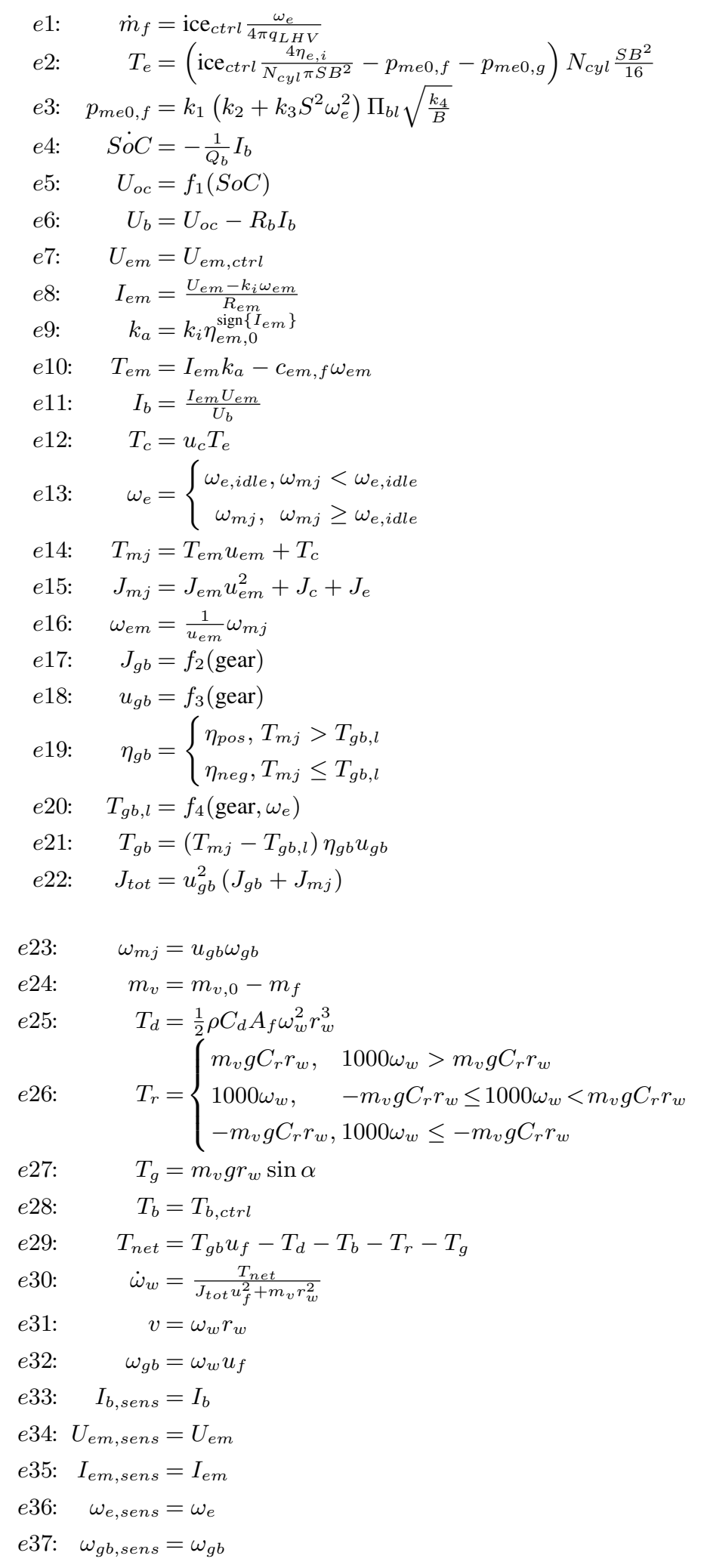

\section{B. Residual generators}

1) Same tests in ICDS and MCDS: Test 1:

$$
\begin{gathered}
\mathcal{C}_{T 1}=\left\{\left(\left\{U_{e m}\right\},\left\{e_{7}\right\}\right)\right\} \\
A R R_{T 1}=e_{34}
\end{gathered}
$$

Test 2:

$\mathcal{C}_{T 2}=\left\{\left(\left\{I_{b}\right\},\left\{e_{33}\right\}\right),\left(\left\{I_{e m}\right\},\left\{e_{35}\right\}\right),\left(\left\{\omega_{g b}\right\},\left\{e_{37}\right\}\right)\right.$, $\left(\left\{u_{g b}\right\},\left\{e_{18}\right\}\right),\left(\left\{\omega_{m j}\right\},\left\{e_{23}\right\}\right),\left(\left\{\omega_{e m}\right\},\left\{e_{16}\right\}\right),\left(\{S o C\},\left\{e_{4}\right\}\right)$, $\left.\left(\left\{U_{o c}\right\},\left\{e_{5}\right\}\right),\left(\left\{U_{b}\right\},\left\{e_{6}\right\}\right),\left(\left\{U_{e m}\right\},\left\{e_{8}\right\}\right)\right\}$

$$
A R R_{T 2}=e_{11}
$$

\section{2) ICDS: Test 3:}

$\mathcal{C}_{T 3, I}=\left\{\left(\left\{\omega_{e}\right\},\left\{e_{36}\right\}\right),\left(\left\{\omega_{g b}\right\},\left\{e_{37}\right\}\right),\left(\left\{u_{g b}\right\},\left\{e_{18}\right\}\right)\right.$, $\left(\left\{\omega_{m j}\right\},\left\{e_{23}\right\}\right),\left(\left\{\omega_{e m}\right\},\left\{e_{16}\right\}\right),\left(\left\{U_{e m}\right\},\left\{e_{7}\right\}\right),\left(\left\{I_{e m}\right\},\left\{e_{8}\right\}\right)$, $\left(\left\{k_{a}\right\},\left\{e_{9}\right\}\right),\left(\left\{T_{e m}\right\},\left\{e_{10}\right\}\right),\left(\left\{p_{m e 0, f}\right\},\left\{e_{3}\right\}\right),\left(\left\{T_{e}\right\},\left\{e_{2}\right\}\right)$, $\left(\left\{m_{f}\right\},\left\{e_{1}\right\}\right),\left(\left\{J_{g b}\right\},\left\{e_{17}\right\}\right),\left(\left\{J_{m j}\right\},\left\{e_{15}\right\}\right),\left(\left\{J_{t o t}\right\},\left\{e_{22}\right\}\right)$, $\left(\left\{T_{g b, l}\right\},\left\{e_{20}\right\}\right),\left(\left\{T_{c}\right\},\left\{e_{12}\right\}\right),\left(\left\{T_{m j}\right\},\left\{e_{14}\right\}\right),\left(\left\{\eta_{g b}\right\},\left\{e_{19}\right\}\right)$, $\left(\left\{m_{v}\right\},\left\{e_{24}\right\}\right),\left(\left\{T_{g}\right\},\left\{e_{27}\right\}\right),\left(\left\{T_{g b}\right\},\left\{e_{21}\right\}\right),\left(\left\{T_{b}\right\},\left\{e_{28}\right\}\right)$, $\left.\left(\left\{T_{d}, T_{r}, T_{n e t}, \omega_{w}\right\},\left\{e_{25}, e_{26}, e_{29}, e_{30}\right\}\right)\right\} \quad$ (49a)

$$
A R R_{T 3, I}=e_{32}
$$

\section{Test 4:}

$\mathcal{C}_{T 4, I}=\left(\left\{I_{b}\right\},\left\{e_{33}\right\}\right),\left\{\left(\left\{U_{e m}\right\},\left\{e_{34}\right\}\right),\left(\left\{\omega_{e}\right\},\left\{e_{36}\right\}\right)\right.$, $\left(\left\{\omega_{g b}\right\},\left\{e_{37}\right\}\right),\left(\left\{u_{g b}\right\},\left\{e_{18}\right\}\right),\left(\left\{\omega_{m j}\right\},\left\{e_{23}\right\}\right),\left(\left\{\omega_{e m}\right\},\left\{e_{16}\right\}\right)$, $\left(\{S o C\},\left\{e_{4}\right\}\right),\left(\left\{U_{o c}\right\},\left\{e_{5}\right\}\right),\left(\left\{U_{b}\right\},\left\{e_{6}\right\}\right),\left(\left\{I_{e m}\right\},\left\{e_{11}\right\}\right)$, $\left(\left\{k_{a}\right\},\left\{e_{9}\right\}\right),\left(\left\{T_{e m}\right\},\left\{e_{10}\right\}\right),\left(\left\{p_{m e 0, f}\right\},\left\{e_{3}\right\}\right),\left(\left\{T_{e}\right\},\left\{e_{2}\right\}\right)$, $\left(\left\{m_{f}\right\},\left\{e_{1}\right\}\right),\left(\left\{J_{g b}\right\},\left\{e_{17}\right\}\right),\left(\left\{J_{m j}\right\},\left\{e_{15}\right\}\right),\left(\left\{J_{t o t}\right\},\left\{e_{22}\right\}\right)$, $\left(\left\{T_{g b, l}\right\},\left\{e_{20}\right\}\right),\left(\left\{T_{c}\right\},\left\{e_{12}\right\}\right),\left(\left\{T_{m j}\right\},\left\{e_{14}\right\}\right),\left(\left\{\eta_{g b}\right\},\left\{e_{19}\right\}\right)$, $\left(\left\{m_{v}\right\},\left\{e_{24}\right\}\right),\left(\left\{T_{g}\right\},\left\{e_{27}\right\}\right),\left(\left\{T_{g b}\right\},\left\{e_{21}\right\}\right),\left(\left\{T_{b}\right\},\left\{e_{28}\right\}\right)$, $\left.\left(\left\{T_{d}, T_{r}, T_{n e t}, \omega_{w}\right\},\left\{e_{25}, e_{26}, e_{29}, e_{30}\right\}\right)\right\} \quad$ (50a)

$$
A R R_{T 4, I}=e_{32}
$$

\section{3) MCDS: Test 3:}

$\mathcal{C}_{T 3, M}=\left\{\left(\left\{\omega_{e}\right\},\left\{e_{36}\right\}\right),\left(\left\{\omega_{g b}\right\},\left\{e_{37}\right\}\right),\left(\left\{u_{g b}\right\},\left\{e_{18}\right\}\right)\right.$, $\left(\left\{\omega_{m j}\right\},\left\{e_{23}\right\}\right),\left(\left\{\omega_{e m}\right\},\left\{e_{16}\right\}\right),\left(\left\{U_{e m}\right\},\left\{e_{7}\right\}\right),\left(\left\{I_{e m}\right\},\left\{e_{8}\right\}\right)$, $\left(\left\{k_{a}\right\},\left\{e_{9}\right\}\right),\left(\left\{T_{e m}\right\},\left\{e_{10}\right\}\right),\left(\left\{p_{m e 0, f}\right\},\left\{e_{3}\right\}\right),\left(\left\{T_{e}\right\},\left\{e_{2}\right\}\right)$, $\left(\left\{m_{f}\right\},\left\{e_{1}\right\}\right),\left(\left\{J_{g b}\right\},\left\{e_{17}\right\}\right),\left(\left\{J_{m j}\right\},\left\{e_{15}\right\}\right),\left(\left\{J_{t o t}\right\},\left\{e_{22}\right\}\right)$, $\left(\left\{T_{g b, l}\right\},\left\{e_{20}\right\}\right),\left(\left\{T_{c}\right\},\left\{e_{12}\right\}\right),\left(\left\{T_{m j}\right\},\left\{e_{14}\right\}\right),\left(\left\{\eta_{g b}\right\},\left\{e_{19}\right\}\right)$, $\left(\left\{m_{v}\right\},\left\{e_{24}\right\}\right),\left(\left\{T_{g}\right\},\left\{e_{27}\right\}\right),\left(\left\{T_{g b}\right\},\left\{e_{21}\right\}\right),\left(\left\{T_{b}\right\},\left\{e_{28}\right\}\right)$, $\left.\left(\left\{\omega_{w}\right\},\left\{e_{32}\right\}\right),\left(\left\{T_{d}\right\},\left\{e_{25}\right\}\right),\left(\left\{T_{r}\right\},\left\{e_{26}\right\}\right),\left(\left\{T_{n e t}\right\},\left\{e_{29}\right\}\right)\right\}$

$$
A R R_{T 3, M}=e_{30}
$$

Test 4:

$\mathcal{C}_{T 4, M}=\left(\left\{I_{b}\right\},\left\{e_{33}\right\}\right),\left\{\left(\left\{U_{e m}\right\},\left\{e_{34}\right\}\right),\left(\left\{\omega_{e}\right\},\left\{e_{36}\right\}\right)\right.$, $\left(\left\{\omega_{g b}\right\},\left\{e_{37}\right\}\right),\left(\left\{u_{g b}\right\},\left\{e_{18}\right\}\right),\left(\left\{\omega_{m j}\right\},\left\{e_{23}\right\}\right),\left(\left\{\omega_{e m}\right\},\left\{e_{16}\right\}\right)$, $\left(\{S o C\},\left\{e_{4}\right\}\right),\left(\left\{U_{o c}\right\},\left\{e_{5}\right\}\right),\left(\left\{U_{b}\right\},\left\{e_{6}\right\}\right),\left(\left\{I_{e m}\right\},\left\{e_{11}\right\}\right)$, $\left(\left\{k_{a}\right\},\left\{e_{9}\right\}\right),\left(\left\{T_{e m}\right\},\left\{e_{10}\right\}\right),\left(\left\{p_{m e 0, f}\right\},\left\{e_{3}\right\}\right),\left(\left\{T_{e}\right\},\left\{e_{2}\right\}\right)$, $\left(\left\{m_{f}\right\},\left\{e_{1}\right\}\right),\left(\left\{J_{g b}\right\},\left\{e_{17}\right\}\right),\left(\left\{J_{m j}\right\},\left\{e_{15}\right\}\right),\left(\left\{J_{t o t}\right\},\left\{e_{22}\right\}\right)$, $\left(\left\{T_{g b, l}\right\},\left\{e_{20}\right\}\right),\left(\left\{T_{c}\right\},\left\{e_{12}\right\}\right),\left(\left\{T_{m j}\right\},\left\{e_{14}\right\}\right),\left(\left\{\eta_{g b}\right\},\left\{e_{19}\right\}\right)$, $\left(\left\{m_{v}\right\},\left\{e_{24}\right\}\right),\left(\left\{T_{g}\right\},\left\{e_{27}\right\}\right),\left(\left\{T_{g b}\right\},\left\{e_{21}\right\}\right),\left(\left\{T_{b}\right\},\left\{e_{28}\right\}\right)$, $\left.\left(\left\{\omega_{w}\right\},\left\{e_{32}\right\}\right),\left(\left\{T_{d}\right\},\left\{e_{25}\right\}\right),\left(\left\{T_{r}\right\},\left\{e_{26}\right\}\right),\left(\left\{T_{n e t}\right\},\left\{e_{29}\right\}\right)\right\}$

$$
A R R_{T 4, M}=e_{30}
$$




\section{REFERENCES}

[1] M. Blanke, M. Kinnaert, J. Lunze, and M. Staroswiecki, Diagnosis and Fault-Tolerant Control, 2nd ed. Springer, 2006.

[2] S. Gentil, J. Montmain, and C. Combastel, "Combining fdi and ai approaches within causal-model-based diagnosis," IEEE Trans. on SMC - Part B, vol. 34, no. 5, pp. 2207 -2221, oct. 2004.

[3] M.-O. Cordier, P. Dague, F. Levy, J. Montmain, M. Staroswiecki, and L. Trave-Massuyes, "Conflicts versus analytical redundancy relations: a comparative analysis of the model based diagnosis approach from the artificial intelligence and automatic control perspectives," IEEE Trans. on SMC - Part B, vol. 34, no. 5, pp. 2163 -2177, oct. 2004.

[4] E. Page, "Continuous inspection schemes," Biometrika, vol. 41, no. 1-2, pp. 100-115, June 1954.

[5] L. Guzzella and A. Sciarretta, Vehicle Propulsion System, Introduction to Modeling and Optimization, 2nd ed. Zürich: Springer Verlag, 2007.

[6] M. Staroswiecki and P. Declerck, "Analytical redundancy in non-linear interconnected systems by means of structural analysis," in Proceedings of IFAC AIPAC'89, Nancy, France, 1989, pp. 51-55.

[7] C. Svärd and M. Nyberg, "Residual generators for fault diagnosis using computation sequences with mixed causality applied to automotive systems," IEEE Trans. on SMC - Part A, vol. 40, no. 6, pp. 1310-1328, 2010.

[8] E. Chow and A. Willsky, "Analytical redundancy and the design of robust failure detection systems," Automatic Control, IEEE Transactions on, vol. 29, no. 7, pp. 603 - 614, July 1984.

[9] M. Staroswiecki and G. Comtet-Varga, "Analytical redundancy relations for fault detection and isolation in algebraic dynamic systems," Automatica, vol. 37, no. 5, pp. 687 - 699, 2001.

[10] R. Isermann, Fault Diagnosis Systems - An Introduction from fault Detection to Fault Tolerance. Springer Verlag, 2006.

[11] P. M. Frank, "Enhancement of robustness in observer-based fault detection," Int. J. of Control, vol. 59, no. 4, pp. 955-981, 1994.

[12] M. Krysander, J. Åslund, and M. Nyberg, "An efficient algorithm for finding minimal over-constrained sub-systems for model-based diagnosis," IEEE Trans. on SMC - Part A, vol. 38, no. 1, 2008.

[13] E. Frisk, A. Bregon, J. Åslund, M. Krysander, B. Pulido, and G. Biswas, "Diagnosability analysis considering causal interpretations for differential constraints," IEEE Trans. on SMC - Part A: Systems and Humans, vol. 42, no. 5, pp. 1216-1229, September 2012.

[14] J. Fredriksson, J. Larsson, J. Sjöberg, and P. Krus, "Evaluating hybrid electric and fuel cell vehicles using the capsim simulation environment," in 22nd International Battery, Hybrid and Fuel Cell Electric Vehicle Symposium \& Exposition, Yokohama, Japan, 2006, pp. $1994-2004$.

[15] L. Guzzella and A. Amstutz, "CAE tools for quasi-static modeling and optimization of hybrid powertrains," IEEE Trans. on Vehicular Tech., vol. 48, no. 6, pp. 1762-1769, Nov. 1999.

[16] C. Sundström, "Vehicle level diagnosis for hybrid powertrains," Tech. Rep., 2011, licentiate thesis LiU-TEK-LIC-2011:27, Thesis No. 1488.

[17] C. Sundström, E. Frisk, and L. Nielsen, "Overall monitoring and diagnosis for hybrid vehicle powertrains," in 6th IFAC Symposium on Advances in Automotive Control, Munich, Germany, 2010, pp. 119-124.

[18] G. Rizzoni, L. Guzzella, and B. Baumann, "Unified modeling of hybrid electric vehicle drivetrains," Mechatronics, IEEE/ASME Transactions on, vol. 4, no. 3, pp. 246 -257, Sept. 1999.

[19] T. B. Reddy, Linden's Handbook of Batteries. McGraw-Hill, 2011.

[20] P. Nelson, K. Amine, A. Rousseau, and H. Yomoto, "Advanced lithiumion batteries for plug-in hybrid-electric vehicles," in Proceedings in 23rd International Electric Vehicle Symposium, Anaheim, CA, 2007, pp. 1589-1605.

[21] K. Chau, C. Chan, and C. Liu, "Overview of permanent-magnet brushless drives for electric and hybrid electric vehicles," Industrial Electronics, IEEE Trans. on, vol. 55, no. 6, pp. 2246 -2257, June 2008.

[22] L. Eriksson, "Simulation of a vehicle in longitudinal motion with clutch engagement and release," in IFAC Workshop: Advances in Automotive Control, Karlsruhe, Germany, 2001, pp. 65-70.

[23] G. Katsillis and M. Chantler, "Can dependency-based diagnosis cope with simultaneous equations?" in 8th International Workshop on Principles of Diagnosis (DX-97), Le Moint Saint Michel, France, 1997, pp. 51-59.

[24] A. R. Hambley, Electrical Engineering, principles and applications, 3rd ed. Upper Sadle River: Pearson Education, 2005.

[25] L. Barford, E. Manders, G. Biswas, P. Mosterman, V. Ram, and J. Barnett, "Derivative estimation for diagnosis," HP Laboratories Palo Alto, Tech. Rep., 1999, HPL-1999-18.
[26] E. Frisk and J. Åslund, "Lowering orders of derivatives in non-linear residual generation using realization theory," Automatica, vol. 41, no. 10, pp. 1799-1807, 2005.

[27] E. Frisk and M. Nyberg, "A minimal polynomial basis solution to residual generation for fault diagnosis in linear systems," Automatica, vol. 37, no. 9, pp. 1417-1424, September 2001.

[28] F. Gustafsson, Adaptive filtering and change detection. John Wiley \& Sons, 2000.

[29] M. Krysander and E. Frisk, "Sensor placement for fault diagnosis," IEEE Trans. on SMC - Part A, vol. 38, no. 6, pp. 1398-1410, 2008.

[30] M. Krysander, F. Heintz, J. Roll, and E. Frisk, "FlexDx: A reconfigurable diagnosis framework," Engineering Applications of Artificial Intelligence, vol. 23, no. 8, pp. 1303-1313, October 2010. 